\title{
EARLY CAREER RESEARCHERS
}

\section{Nutrigenetics and blood pressure optimization - focus on fat intake and fatty acid desaturase function}

\author{
MANJA M. ZEC \\ School of Nutritional Sciences and Wellness, University of Arizona, Tucson AZ, United States
}

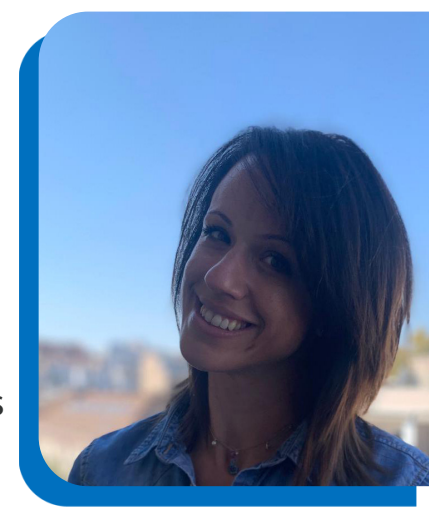

DOI:10.30824/2112-12

Precision nutrition based on targeted approaches such as nutrigenetics, has become an essential tool in lifestyle interventional strategies for managing cardiometabolic risk factors. Hypertension management remains instrumental in preventing cardiovascular disease, however, there is a huge gap between standard pharmacological approaches for optimizing blood pressure and lifestyle strategies for preventing its raise. Dietary actions based on salt intake reduction and prudent fat consumption are pivotal in sustaining optimal blood pressure levels, regardless of demographics or other health contexts. Customizing adequate fat intake gains increasing interest in light of genetic variability associated with fat and fatty acid metabolism and subsequent health implications.

Fatty acid desaturase gene cluster (including FADS-1 and -2 genes) resides on human chromosome 11 and encodes for the desaturase enzymes which catalyze endogenous conversion of essential fatty acids from foods towards highly unsaturated omega- 6 and omega- 3 plasma and tissue products. The omega-3 commonly refer to eicosapentaenoic acid (EPA, C20:5 n-3) and docosahexaenoic acid (DHA, C22:6 n-3), considered for their inflammation-resolving, endothelium-stabilizing and anti-thrombotic effects. Early research endeavors demonstrated the association between the FADS genes variability and levels of long-chain polyunsaturated fatty acids (PUFA) in plasma ${ }^{1}$, then dyslipidemia factors such as high-density lipoprotein cholesterol and triglycerides 2 . A recent study indicated that the single nucleotide polymorphism in FADS-2 gene might be linked with diastolic blood pressure in men only and depending on the adherence to a Dietary Approach to Stop Hypertension pattern ${ }^{3}$.

Several studies indicate a connection between longchain PUFA profiles in plasma, and vascular function. Specifically in black Africans undergoing nutritional transition, plasma omega-6 PUFA were beneficially linked with blood pressure ${ }^{4}$, while the inverse link was observed for DHA in Chinese, community dwelling subjects ${ }^{5}$.
Importantly, certain populations, such as black Africans, have pronounced levels of long-chain PUFA in plasma ${ }^{4}$, which could at least partly be explained by the ethnicspecific and race-specific distribution of FADS haplotype reflected in different metabolic conversion ${ }^{6}$. There is thus a sound rationalization to presume that a variability in FADS human genetic region might also be linked with endothelial stability and vascular function, and future studies are urged to assess the link between the variable FADS region, fat intake, FADS-dietary interactions, and vascular implications, specifically systolic and diastolic blood pressure. Accounting for the population-specific fat metabolism features in customizing public health and personalized guidelines and recommendations, might support combating disproportionate disease burden in vulnerable groups, ultimately alleviating cardiovascular disease across world-wide populations.

Previous reports proposed nutrigenetic protocols for implementing FADS genotyping in precision nutrition assessments for personalized dietary and nutritional plans, as a standard of care ${ }^{7}$. Should the FADS genetic information be interpreted as a predisposition towards various amounts of advantageous omega-3 PUFA but also omega-6PUFA in plasma, this might complement the information about the level of individual cardiometabolic risk associated with excessive fat intake. This way the FADS individual genetic signature stands out as a valuable print in customizing personalized plans and appropriate fat intake, for overall wellness and fitness, possibly leveraging blood pressure levels. What's more, previous report encouraged the pharmaco-nutrigenetic approach in balancing blood pressure in subjects already taking anti-hypertensive medication and based on customizing their daily salt intake according to the genetically driven salt-sensitivity phenotype, with a few predictive genetic biomarkers already replicated in several studies ${ }^{8}$. The FADS nutrigenetic evaluation in routine precision nutrition practice remains envisioned strategy for successful blood pressure optimization in pharmaco-nutrigenetic setting, in addition to emerging 
as a promising tool for preventing blood pressure raise across world-wide populations.

\section{REFERENCES}

1.Tanaka, T., Shen, J., Abecasis, G.R., et al., 2009. Genomewide association study of plasma polyunsaturated fatty acids in the InCHIANTI Study. PLoS genetics, 5(1), p.e1000338.

2. Kathiresan, S., Willer, C.J., Peloso, G.M., Demissie, et al. 2009. Common variants at 30 loci contribute to polygenic dyslipidemia. Nature genetics, 41(1), p.56-65.

3. Khodarahmi, M., Nikniaz, L. and Farhangi, M.A., 2021. The Interaction Between Fatty Acid Desaturase-2 (FADS2) rs174583 Genetic Variant and Dietary Quality Indices (DASH and MDS) Constructs Different Metabolic Phenotypes Among Obese Individuals. Frontiers in Nutrition, 8.

4. Zec, M.M., Schutte, A.E., Ricci, C., Baumgartner, J., Kruger, I.M. and Smuts, C.M., 2019. Long-chain polyunsaturated fatty acids are associated with blood pressure and hypertension over 10-years in black South African adults undergoing nutritional transition. Foods, 8(9), p.394.
5. Yang, B., Ding, F., Yan, J., Ye, X.W., Xu, X.L., Wang, F.L., Li, D. and Yu, W., 2016. Exploratory serum fatty acid patterns associated with blood pressure in community-dwelling middle-aged and elderly Chinese. Lipids in health and disease, 15(1), p.1-11.

6. Mathias, R.A., Fu, W., Akey, J.M., Ainsworth, H.C., Torgerson, D.G., Ruczinski, I., Sergeant, S., Barnes, K.C. and Chilton, F.H., 2012. Adaptive evolution of the FADS gene cluster within Africa. Plos One. 2012;7(9), e44926

7. Chilton, F.H., Dutta, R., Reynolds, L.M., Sergeant, S., Mathias, R.A. and Seeds, M.C., 2017. Precision nutrition and omega-3 polyunsaturated fatty acids: A case for personalized supplementation approaches for the prevention and management of human diseases. Nutrients, 9(11), p.1165.

8. Dong, O.M., 2018. Excessive dietary sodium intake and elevated blood pressure: a review of current prevention and management strategies and the emerging role of pharmaconutrigenetics. BMJ nutrition, prevention \& health, 1(1), p.7.

a:care

\section{"Medication adherence: What you can do with the medical challenge that starts after the consultation"}

a:care Congress 2021

\section{Did you miss it?}

The first congress exploring medication adherence through behavioral science was held online in October and was attended by almost 10,000 healthcare professionals from around the world.

Presentations from global experts, including Professor John Weinman of King's College

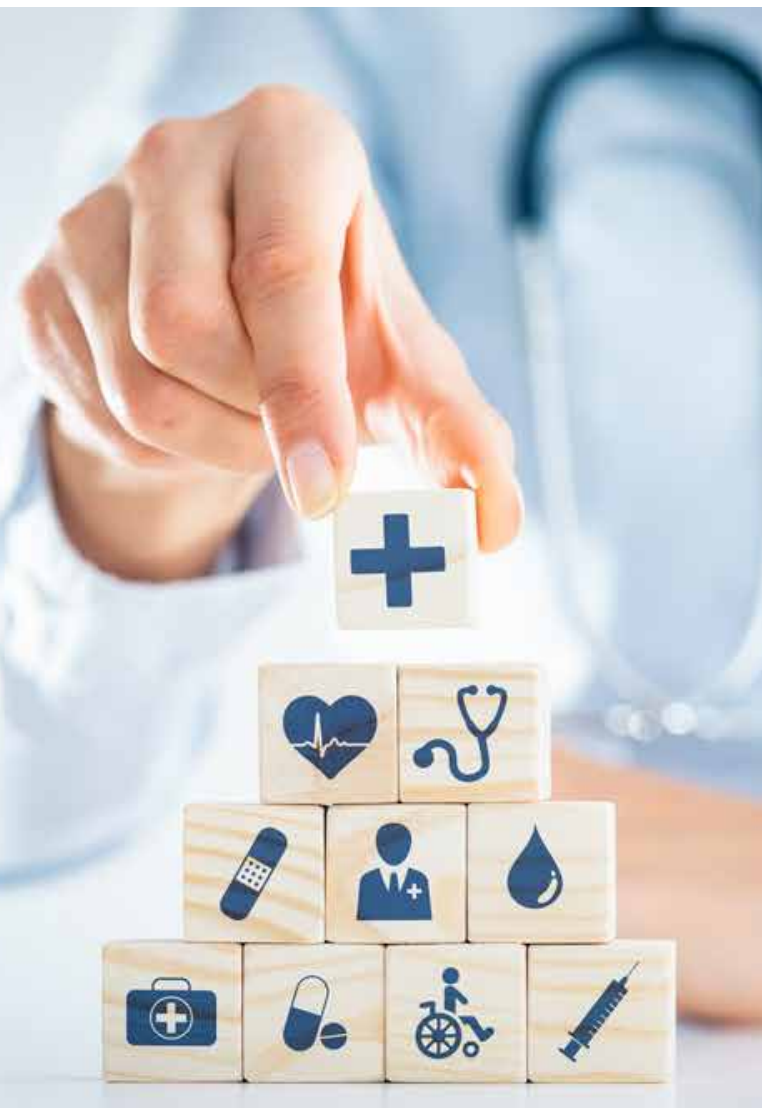
London, were recorded and are now available for you to watch at acarepro.abbott.com 